\title{
Magnitude of potentially inappropriate prescribing in Germany among older patients with generalized anxiety disorder Ariel Berger ${ }^{1}$, Marko Mychaskiw ${ }^{2}$, Ellen Dukes ${ }^{3}$, John Edelsberg${ }^{1}$ and Gerry Oster*1
}

\author{
Address: ${ }^{1}$ Policy Analysis, Inc, Brookline, USA, ${ }^{2}$ Worldwide Medical and Outcomes Research, Pfizer, Inc, New York City, USA and ${ }^{3}$ Global \\ Outcomes Research, Pfizer, Inc New York City, USA \\ Email: Ariel Berger - aberger@pai2.com; Marko Mychaskiw - Marko.Mychaskiw@pfizer.com; Ellen Dukes - Ellen.Dukes@pfizer.com; \\ John Edelsberg - edelsberg@pai2.com; Gerry Oster* - goster@pai2.com \\ * Corresponding author
}

Published: 27 July 2009

BMC Geriatrics 2009, 9:31 doi:10.1|86/|47|-23|8-9-3|

This article is available from: http://www.biomedcentral.com/|47|-23/8/9/3I

(C) 2009 Berger et al; licensee BioMed Central Ltd.

This is an Open Access article distributed under the terms of the Creative Commons Attribution License (http://creativecommons.org/licenses/by/2.0), which permits unrestricted use, distribution, and reproduction in any medium, provided the original work is properly cited.
Received: 6 February 2009

Accepted: 27 July 2009

\begin{abstract}
Background: Several medications commonly used to treat generalized anxiety disorder (GAD) have been designated "potentially inappropriate" for use in patients aged $\geq 65$ years because their risks may outweigh their potential benefits. The actual extent of use of these agents in clinical practice is unknown, however.

Methods: Using a database with information from encounters with general practitioners (GP) in Germany, we identified all patients, aged $\geq 65$ years, with any GP office visits or dispensed prescriptions with a diagnosis of GAD (ICD-I0 diagnosis code F4I.I) between I0/I/2003 and 9/30/ 2004 ("GAD patients"). Among GAD-related medications (including benzodiazepines, tricyclic antidepressants [TCAs], selective serotonin reuptake inhibitors, venlafaxine, hydroxyzine, buspirone, pregabalin, and trifluoperazine), long-acting benzodiazepines, selected short-acting benzodiazepines at relatively high dosages, selected TCAs, and hydroxyzine were designated "potentially inappropriate" for use in patients aged $\geq 65$ years, based on published criteria.
\end{abstract}

Results: A total of 975 elderly patients with GAD were identified. Mean age was 75 years, and $72 \%$ were women; $29 \%$ had diagnoses of comorbid depression. Forty percent of study subjects received potentially inappropriate agents - most commonly, bromazepam (10\% of all subjects), diazepam (9\%), doxepin (7\%), amitriptyline (5\%), and lorazepam (5\%). Twenty-three percent of study subjects received long-acting benzodiazepines, $10 \%$ received short-acting benzodiazepines at relatively high doses, and $12 \%$ received TCAs designated as potentially inappropriate.

Conclusion: GPs in Germany often prescribe medications that have been designated as potentially inappropriate to their elderly patients with GAD - especially those with comorbid depressive disorders. Further research is needed to ascertain whether there are specific subgoups of elderly patients with GAD for whom the benefits of these medications outweigh their risks.

\section{Background}

Generalized anxiety disorder (GAD) is a chronic condition that is characterized by persistent worry or anxiety that occurs more days than not over a period of at least six months [1]. The condition is frequently difficult to diagnose because of the variety of clinical presentations and 
the common occurrence of comorbid medical or other psychiatric conditions. Lifetime prevalence has been estimated to be between $4 \%$ and $6 \%$ [2]; the disease is more common among women than men. GAD is the most common anxiety disorder among patients presenting to primary care physicians $[3,4]$.

Several different types of medications are often used to treat GAD - specifically, benzodiazepines (e.g., flurazepam, diazepam, chlordiazepoxide), buspirone, tricyclic antidepressants (TCAs) (e.g., amitriptyline, imipramine, doxepin, opipramol), selective serotonin reuptake inhibitors (SSRIs) (e.g., paroxetine. escitalopram), and venlafaxine (a selective serotonin and norepinephrine reuptake inhibitor) [5-7]. Among these available therapies, benzodiazepines have long been the mainstay of pharmacologic treatment for GAD. While effective, benzodiazepines are associated with excessive sedation and motor impairment [8]; their long-term use is also associated with a risk of physical dependence as well as withdrawal when therapy is discontinued [6]. In one study comparing 4554 persons prescribed benzodiazepines with 13,662 persons receiving other (i.e., nonbenzodiazepine) medications who were matched on age, sex, and calendar month in which therapy was initiated, Oster and colleagues found that patients in the former group had a $15 \%$ higher risk of an accident-related medical event; those who filled three or more prescriptions for benzodiazepines had a 30\% higher risk compared with those who filled only one such prescription [9].

An expert panel convened by Beers in 1991 developed explicit criteria for identifying medication use among nursing home residents that was potentially inappropriate [10]. Recognizing that these criteria were developed specifically for a nursing home population, Beers convened another expert panel in 1997 to develop criteria applicable to the entire population of older persons ( $\geq 65$ years); the resulting criteria designated some of the drugs used to treat GAD (benzodiazepines, amitriptyline, doxepin) as potentially inappropriate for use in persons aged $\geq 65$ years [11]. The panel compiled its list of potentially inappropriate medications without regard to diagnosis or place of residence, and sought to include only those agents whose ". . . potential for adverse outcomes is greater than the potential for benefit" [11].

While well-known and extensively cited, the Beers' criteria have been criticized as not providing a sufficient basis for identifying inappropriate prescribing, as they are not indication-specific [12]. A subsequent expert panel convened by Zhan et al. classified 33 medications on the Beers' list alternatively as always to be avoided, rarely appropriate, and appropriate for some indications [13]. Among drugs that are sometimes used to treat GAD, flurazepam was designated as "always to be avoided"; chlordiazepoxide and diazepam were designated as "rarely appropriate"; and amitriptyline and doxepin, "appropriate for some indications".

In their update of the Beers' criteria, Fick et al. designated flurazepam, amitriptyline, chlordiazepoxide, doxepin, and anything other than low doses of short-acting benzodiazepines (e.g., >3 mg lorazepam) as potentially inappropriate for use in older patients; adverse outcomes for all such medications were deemed by the authors to be of high (versus low) severity [14].

Despite their limitations, the 1997 Beers' criteria have been widely used by researchers to identify potential medication risks [13,15-19]. An epidemiologic study of noninstitutionalized persons who participated in the 1987 US National Medical Expenditure Survey reported that 23.5\% of those aged $\geq 65$ years received at least one of the 20 medications on the Beers' list [20]. Zhan et al. applied their revised list to persons participating in the 1996 US Medical Expenditure Panel Survey and reported that $21.3 \%$ received drugs that were potentially - albeit not necessarily - inappropriate, $2.6 \%$ received medications that should always be avoided, and $9.1 \%$ received drugs that were rarely appropriate; $3.4 \%$ of those aged $>65$ years had received amitriptyline [13].

Recently, an examination of 2707 older home-care patients from eight countries across Europe found that $19.8 \%$ received at least one medication designated as potentially inappropriate; in multivariate analysis, use of anxiolytics was associated with a twofold increase in the likelihood of receiving potentially inappropriate medications [21]. Some of the most commonly used, potentially inappropriate medications in this study were benzodiazepines (diazepam [3.1\% of patients received such therapy] and chlordiazepoxide [0.6\%]) and tricyclic antidepressants (amitriptyline [1.4\%]) - agents often used for the treatment of GAD.

Although older adults with GAD would appear to often receive potentially inappropriate medications, the actual extent of such use in clinical practice is unknown. Moreover, the generalizability of earlier findings - based largely on US data - to other countries is unknown. In this study, we examine the magnitude of exposure of patients aged $\geq 65$ years with GAD to potentially inappropriate medications in Germany, a large European country in which observed patterns of treatment of GAD may possibly be reflective of those throughout Europe.

\section{Methods}

Data were obtained from the IMS MediPlus - Disease Analyzer database; a detailed description of the database 
and its research capabilities may be found elsewhere [22]. The database is longitudinal in nature, and provides patient-level information on consultations, diagnoses, and treatments from over 900 GP practices throughout Germany, comprising more than 4.2 million patient records and 75 million prescriptions over a 10-year period. The database is compiled by sampling practices throughout Germany, and is designed to be representative of the general population in Germany. All patient identifiers in the database are fully encrypted.

Information in the IMS MediPlus - Disease Analyzer database includes date of service, diagnoses (in ICD-10 format), actions taken (e.g., referrals to other providers [i.e., specialists], dispensing of sick notes [physician-excused absences from work]), and medications dispensed, including the dispensing date, the quantity dispensed, the number of days of therapy supplied, and the associated diagnosis (available for about $60 \%$ of all prescription records). Selected demographic information is also available, including patient age and gender. All patient-level data can be arrayed chronologically to provide a detailed, longitudinal profile of all medical and pharmacy services rendered by participating GPs. Because this study was retrospective in nature, used completely anonymized data, and did not involve patient contact, institutional review board (IRB) approval was not sought. The database for this study encompassed the period, October 1, 2003 through September 30, 2004 ("study period").

The study sample consisted of all patients with any GP office visits or dispensed prescriptions with a diagnosis of GAD (ICD-10 diagnosis code F41.1) during the study period. Persons aged $<65$ years as of their first-noted encounter for GAD were excluded from the study sample. All GP encounters were then compiled for all subjects over the one-year period of study.

The prevalence of a number of medically attended comorbidities (i.e., noted by GPs during office or clinic visits) was examined for patients in the study sample, including: (1) neoplasms; (2) anemia and other blood antibody disorders; (3) diabetes; (4) circulatory system disorders; (5) respiratory system disorders; (6) eye, nose, and throat disorders; (7) digestive system disorders; (8) painful neuropathic disorders (e.g., diabetic peripheral neuropathy, post-herpetic neuralgia, causalgia, neuropathic back pain); (9) musculoskeletal system disorders; (10) symptom, signs, ill-defined conditions; (11) somatoform disorders; (12) neurasthenia; (13) substance use disorders; and (14) sleep disorders. Patients were deemed to have these conditions if they had any encounters during the study period with the corresponding diagnosis code(s).

The numbers of patients receiving various medications often used to treat GAD ("GAD-related medications") were tabulated. Medications (and corresponding dosages, if relevant [see below]) were deemed "potentially inappropriate" based on their inclusion in Beers' 1997 criteria and/or in subsequent updates to these criteria $[11,13,14]$. Medication regimens designated as potentially inappropriate were as follows: (1) alprazolam (>2 mg daily); (2) amitriptyline; (3) chlorazepate; (4) chlordiazepoxide; (5) diazepam; (6) doxepin; (7) flurazepam; (8) halazepam; (9) lorazepam (>3 mg daily); (10) oxazepam (>60 mg daily); (11) temazepam (>15 mg daily); (12) triazolam ( $>0.25 \mathrm{mg}$ daily); and (13) zolpidem ( $>5 \mathrm{mg}$ daily) [Table $1]$. Daily dose was calculated using information in the database; in instances where such information was missing, daily dose was assumed equivalent to the modal value from all other prescriptions for the same product with non-missing values.

Medication receipt was ascertained for the overall study sample, as well as within strata defined on the basis of age, sex, and selected comorbidities (e.g., depression, neoplasms, respiratory disorders). The statistical significance of differences within strata was ascertained using chisquare tests and Fisher's exact tests, as appropriate. All analyses were conducted using PC-SAS ${ }^{\varpi}$ v.9.1 [23].

\section{Results}

The study sample consisted of 975 patients, aged $\geq 65$ years, with diagnoses of GAD; mean $( \pm \mathrm{SD})$ age was 75.0 $( \pm 7.3)$ years, and $71.6 \%$ were women [Table 2]. Twentyfour percent of study subjects had at least one encounter during the study period at which a diagnosis of another anxiety disorder was noted; $29.2 \%$ had at least one encounter resulting in a diagnosis of depression. The prevalence of other medically attended comorbidities was high, including circulatory disorders $(87.5 \%)$, digestive disorders (56.0\%), respiratory disorders (42.5\%), musculoskeletal disorders $(69.5 \%)$, painful neuropathies (28.8\%), and sleep disorders (24.7\%). Ninety-eight percent of patients had at least one comorbidity, and $87.0 \%$ had three or more.

A total of 607 study subjects (62.3\%) had received one or more GAD-related medications (both those deemed potentially inappropriate and all others) from their GPs most commonly, benzodiazepines (43.7\%), including both short-acting $(24.8 \%)$ and long-acting $(23.1 \%)$ formulations, and TCAs (25.6\%) [Table 3]. Nine percent of patients received SSRIs, and $0.9 \%$ received venlafaxine.

About forty percent of study subjects - or two-thirds of patients $(64.6 \%)$ with evidence of receipt of GAD-related medications - were dispensed at least one therapy considered potentially inappropriate for use in the elderly. Ten percent of patients received short-acting benzodiazepines at dosages that rendered their use potentially inappropriate, $23.1 \%$ received long-acting benzodiazepines, and 
Table I: Medications for treatment of generalized anxiety disorder

\begin{tabular}{|c|c|}
\hline Medication Type & Comment \\
\hline \multicolumn{2}{|l|}{ Benzodiazepines } \\
\hline \multicolumn{2}{|l|}{ Short-acting } \\
\hline Alprazolam & $>2 \mathrm{mg} / \mathrm{d}$ deemed potentially inappropriate \\
\hline \multicolumn{2}{|l|}{ Bromazepam } \\
\hline Lorazepam & $>3 \mathrm{mg} / \mathrm{d}$ deemed potentially inappropriate \\
\hline \multicolumn{2}{|l|}{ Lormetazepam } \\
\hline \multicolumn{2}{|l|}{ Nitrazepam } \\
\hline Oxazepam & $>60 \mathrm{mg} / \mathrm{d}$ deemed potentially inappropriate \\
\hline Temazepam & $>15 \mathrm{mg} / \mathrm{d}$ deemed potentially inappropriate \\
\hline \multicolumn{2}{|l|}{ Tetrazepam } \\
\hline Triazolam & $>0.25 \mathrm{mg} / \mathrm{d}$ deemed potentially inappropriate \\
\hline Zolpidem & $>5 \mathrm{mg} / \mathrm{d}$ deemed potentially inappropriate \\
\hline \multicolumn{2}{|l|}{ Long-acting } \\
\hline Chlordiazepoxide & All long-acting benzodiazepines deemed potentially inappropriate \\
\hline Chlorazepate & All long-acting benzodiazepines deemed potentially inappropriate \\
\hline Clobazam & All long-acting benzodiazepines deemed potentially inappropriate \\
\hline Clonazepam & All long-acting benzodiazepines deemed potentially inappropriate \\
\hline Diazepam & All long-acting benzodiazepines deemed potentially inappropriate \\
\hline Flunitrazepam & All long-acting benzodiazepines deemed potentially inappropriate \\
\hline Flurazepam & All long-acting benzodiazepines deemed potentially inappropriate \\
\hline Halazepam & All long-acting benzodiazepines deemed potentially inappropriate \\
\hline Medazepam & All long-acting benzodiazepines deemed potentially inappropriate \\
\hline Nordazepam & All long-acting benzodiazepines deemed potentially inappropriate \\
\hline Prezepam & All long-acting benzodiazepines deemed potentially inappropriate \\
\hline \multicolumn{2}{|l|}{ Tricyclic antidepressants } \\
\hline Amitriptyline & Deemed potentially inappropriate \\
\hline \multicolumn{2}{|l|}{ Amitriptylinoxide } \\
\hline \multicolumn{2}{|l|}{ Clomipramine } \\
\hline \multicolumn{2}{|l|}{ Dibenzepin } \\
\hline \multicolumn{2}{|l|}{ Desipramine } \\
\hline Doxepin & Deemed potentially inappropriate \\
\hline \multicolumn{2}{|l|}{ Imipramine } \\
\hline \multicolumn{2}{|l|}{ Maprotiline } \\
\hline \multicolumn{2}{|l|}{ Nortiptyline } \\
\hline \multicolumn{2}{|l|}{ Opipramol } \\
\hline \multicolumn{2}{|l|}{ Trimipramine } \\
\hline \multicolumn{2}{|c|}{ Selective serotonin reuptake inhibitor } \\
\hline \multicolumn{2}{|c|}{ Escitalopram } \\
\hline \multicolumn{2}{|l|}{ Citalopram } \\
\hline \multicolumn{2}{|l|}{ Fluoxetine } \\
\hline \multicolumn{2}{|l|}{ Fluvoxamine } \\
\hline \multicolumn{2}{|l|}{ Paroxetine } \\
\hline \multicolumn{2}{|l|}{ Sertraline } \\
\hline Venlafaxine & \\
\hline Hydroxyzine & Deemed potentially inappropriate \\
\hline Buspirone & \\
\hline Pregabalin & \\
\hline Trifluoperazine & \\
\hline
\end{tabular}

$12.2 \%$ received amitriptyline or doxepin (primarily doxepin).

Receipt of potentially inappropriate therapy did not differ by age (39.1\% for patients aged $65-74$ years vs $41.5 \%$ for patients aged $\geq 75$ years; $\mathrm{p}=0.45)$ or gender $(41.1 \%$ of men vs $37.9 \%$ of women; $p=0.36$ ). Patients with comorbid depression were more likely to receive potentially inappropriate therapy ( $51.6 \%$ vs $35.5 \%$ for those without comorbid depression; $\mathrm{p}<0.01$ ), as were those with digestive system disorders $(43.0 \%$ vs $36.6 \%$ for those without these disorders; $\mathrm{p}=0.04)$, and those with sleep disorders (62.2\% vs $33.0 \%$ for those without these disorders; $\mathrm{p}<$ 0.01 ); receipt of these therapies did not differ significantly for patients with versus without any of the other selected comorbidities. 
Table 2: Demographic and clinical characteristics of study subjects $(\mathbf{N}=975)^{*}$

\begin{tabular}{|c|c|}
\hline Characteristic & \\
\hline \multicolumn{2}{|l|}{ Age, years } \\
\hline $65-74$ & $507(52.0)$ \\
\hline $75-84$ & $365(37.4)$ \\
\hline$\geq 85$ & $103(10.6)$ \\
\hline Mean (SD) & $75.0(7.3)$ \\
\hline Females & $698(71.6)$ \\
\hline Males & $277(28.4)$ \\
\hline \multicolumn{2}{|l|}{ Comorbid conditions } \\
\hline \multicolumn{2}{|l|}{ Anxiety disorders } \\
\hline Panic disorder & $17(1.7)$ \\
\hline OCD & $2(0.2)$ \\
\hline PTSD & $0(0.0)$ \\
\hline \multicolumn{2}{|l|}{ Phobias } \\
\hline Social phobia & $0(0.0)$ \\
\hline Agoraphobia & $0(0.0)$ \\
\hline All other phobias & $10(1.0)$ \\
\hline Any phobia & $10(1.0)$ \\
\hline Other anxiety disorders & $215(22.1)$ \\
\hline Any anxiety disorder (other than GAD) & $237(24.3)$ \\
\hline \multicolumn{2}{|l|}{ Depression disorders } \\
\hline Dysthymic disorder & $10(1.0)$ \\
\hline Adjustment disorder with depression & $4(0.4)$ \\
\hline Bipolar depression & $\mathrm{I}(0.1)$ \\
\hline MDD & $36(3.7)$ \\
\hline Unspecified depression & $256(26.3)$ \\
\hline Any depression & $285(29.2)$ \\
\hline Neoplasms & $158(16.2)$ \\
\hline Anemia and other blood/antibody disorders & $136(13.9)$ \\
\hline Diabetes & $269(27.6)$ \\
\hline Circulatory system disorders & $853(87.5)$ \\
\hline Respiratory system disorders & $414(42.5)$ \\
\hline Eyes, nose, and throat & $262(26.9)$ \\
\hline Digestive system disorders & $546(56.0)$ \\
\hline Painful neuropathic disorders & $281(28.8)$ \\
\hline Musculoskeletal system disorders & $679(69.6)$ \\
\hline Symptoms, signs, ill-defined conditions & $610(62.6)$ \\
\hline Somatoform disorders & $130(13.3)$ \\
\hline Neurasthenia & $39(4.0)$ \\
\hline Substance use disorders & $30(3.1)$ \\
\hline Sleep disorders & $241(24.7)$ \\
\hline \multicolumn{2}{|l|}{ Number of comorbidities } \\
\hline 0 & $17(1.7)$ \\
\hline I & $44(4.5)$ \\
\hline 2 & $66(6.8)$ \\
\hline$\geq 3$ & $848(87.0)$ \\
\hline
\end{tabular}

*Unless otherwise indicated, all values represent number (\%) GAD: Generalized anxiety disorder; OCD: Obsessive-compulsive disorder; PTSD: Post-traumatic stress disorder; MDD: Major depressive disorder

\section{Discussion}

GAD can be difficult to treat, and several different medications - including benzodiazepines, buspirone, TCAs, and SSRIs - are recommended for use in these patients [5-7]. Although these therapies are often of benefit, they also can confer significant risks in older adults. Because of these risks, some of these drugs have been designated potentially inappropriate for use in persons aged 65 years or older.

Using the 1997 Beers criteria [11] and subsequent updates $[13,14]$, we found that four out of every 10 patients - and two out of every three of those who received any GADrelated therapy - received medications that have been designated as potentially inappropriate for use among persons aged 65 years and older. Notably, use of these medications did not differ between the "young" old versus the "old" old (i.e., aged $65-74$ years vs $\geq 75$ years).

Benzodiazepines have long been the mainstay of pharmacologic treatment for GAD, and they were the medication most commonly dispensed among subjects in our study: $44 \%$ of patients in our study received these agents. Paradoxically, they also comprise the majority of agents deemed potentially inappropriate for use in the elderly, due in part to an increased risk of falls, hip fractures, druginduced disorders of cognition, and motor vehicle accidents [9,24-27]. We note that GPs dispensed short-acting benzodiazepines at daily doses deemed potentially inappropriate to $10 \%$ of study subjects; they also dispensed long-acting benzodiazepines - which are deemed potentially inappropriate in older patients regardless of daily dose - to $31 \%$ of patients. About one in 10 patients in our study received TCAs deemed potentially inappropriate either amitriptyline or doxepin - despite the fact that several other TCAs (e.g., nortriptyline) are available with similar efficacy that are better tolerated by older patients. We are unaware of any other study of potentially inappropriate prescribing in patients (of any age) with GAD. Compared with rates of potentially inappropriate prescribing reported among the elderly in general $[13,16-21,28]$, the rates we report are considerably higher. This finding is consistent, however, with the fact that many of the medications that have been designated by some experts as potentially inappropriate in the elderly are also commonly recommended by other experts to treat GAD.

As people age, their ability to metabolize drugs decreases, and receptor sensitivity to the effects of pharmacotherapy changes [29,30]. Unfortunately, older adults are often underrepresented in randomized controlled trials [15], an important source of information for prescribers. Consequently, physicians may sometimes prescribe therapies on presumptions of efficacy and safety based on clinical trial results that are not necessarily generalizable to persons of advanced age. This may explain why we found that so many patients with GAD received medications that were potentially inappropriate. Inappropriate prescribing also may result from the use of inappropriate doses of otherwise age-appropriate medications and/or not prescribing medications that may be of benefit (e.g., not prescribing GAD-related therapies for patients with this condition) [31]. Accordingly, our findings probably underestimate 
Table 3: Numbers of study subjects receiving potentially inappropriate medications for treatment of GAD*

\begin{tabular}{|c|c|c|c|}
\hline Medication & Potentially Inappropriate & Possibly Appropriate & Total \\
\hline \multicolumn{4}{|l|}{ Benzodiazepines } \\
\hline \multicolumn{4}{|l|}{ Short-acting } \\
\hline Alprazolam' & $0(0.0)$ & $29(3.0)$ & $29(3.0)$ \\
\hline Lorazepam² & $50(5.1)$ & $21(2.2)$ & $71(7.3)$ \\
\hline Lormetazepam & $14(1.4)$ & $0(0.0)$ & $14(1.4)$ \\
\hline Oxazepam 3 & $0(0.0)^{\prime}$ & $88(9.0)$ & $88(9.0)$ \\
\hline Temazepam 4 & $8(0.8)$ & $0(0.0)$ & $8(0.8)$ \\
\hline Tetrazepam & $0(0.0)$ & $29(3.0)$ & $29(3.0)$ \\
\hline Triazolam $^{5}$ & $0(0.0)$ & $\mathrm{I}(0.1)$ & $\mathrm{I}(0.1)$ \\
\hline Zolpidem 6 & $32(3.3)$ & $3(0.3)$ & $34(3.5)$ \\
\hline Any of above & $97(9.9)$ & $162(16.6)$ & $242(24.8)$ \\
\hline \multicolumn{4}{|l|}{ Long-acting** } \\
\hline Chlordiazepoxide & $6(0.6)$ & --- & $6(0.6)$ \\
\hline Chlorazepate & II (I.I) & --- & II (I.I) \\
\hline Clobazam & $2(0.2)$ & --- & $2(0.2)$ \\
\hline Clonazepam & $3(0.3)$ & --- & $3(0.3)$ \\
\hline Diazepam & $91(9.3)$ & --- & $91(9.3)$ \\
\hline Flunitrazepam & $7(0.7)$ & --- & $7(0.7)$ \\
\hline Flurazepam & $6(0.6)$ & --- & $6(0.6)$ \\
\hline Halazepam & $0(0.0)$ & --- & $0(0.0)$ \\
\hline Medazepam & $5(0.5)$ & --- & $5(0.5)$ \\
\hline Nordazepam & $2(0.2)$ & --- & $2(0.2)$ \\
\hline Prezepam & $0(0.0)$ & -.-- & $0(0.0)$ \\
\hline Bromazepam & $93(9.5)$ & --- & $93(9.5)$ \\
\hline Nitrazepam & $14(1.4)$ & --- & $14(1.4)$ \\
\hline Midazolam & $0(0.0)$ & --- & $0(0.0)$ \\
\hline Any of above & $225(23.1)$ & --- & $225(23.1)$ \\
\hline Any of above & $298(30.6)$ & $162(16.6)$ & $426(43.7)$ \\
\hline \multicolumn{4}{|l|}{ Tricyclic antidepressants } \\
\hline Amitriptyline & $52(5.3)$ & --- & $52(5.3)$ \\
\hline Amitriptylinoxide & $0(0.0)$ & $3(0.3)$ & $3(0.3)$ \\
\hline Clomipramine & $0(0.0)$ & $4(0.4)$ & $4(0.4)$ \\
\hline Dibenzepin & $0(0.0)$ & $3(0.3)$ & $3(0.3)$ \\
\hline Desipramine & $0(0.0)$ & $0(0.0)$ & $0(0.0)$ \\
\hline Doxepin & $70(7.2)$ & --- & $70(7.2)$ \\
\hline Imipramine & $0(0.0)$ & $0(0.0)$ & $0(0.0)$ \\
\hline Maprotiline & $0(0.0)$ & $4(0.4)$ & $4(0.4)$ \\
\hline Nortriptyline & $0(0.0)$ & $0(0.0)$ & $0(0.0)$ \\
\hline Opipramol & $0(0.0)$ & $105(10.8)$ & $105(10.8)$ \\
\hline Trimipramine & $0(0.0)$ & $29(3.0)$ & $29(3.0)$ \\
\hline Any of above & $119(12.2)$ & $142(14.6)$ & $250(25.6)$ \\
\hline \multicolumn{4}{|c|}{ Selective serotonin reuptake inhibitors } \\
\hline Escitalopram & $0(0.0)$ & $7(0.7)$ & $7(0.7)$ \\
\hline Citalopram & $0(0.0)$ & $40(4.1)$ & $40(4.1)$ \\
\hline Fluoxetine & II (I.I) & $0(0.0)$ & II (I.I) \\
\hline Fluvoxamine & $0(0.0)^{\prime}$ & $3(0.3)$ & $3(0.3)$ \\
\hline Paroxetine & $0(0.0)$ & $16(1.6)$ & $16(1.6)$ \\
\hline Sertraline & $0(0.0)$ & $13(1.3)$ & $13(1.3)$ \\
\hline Any of above & $11(1.1)$ & 77 (7.9) & $86(8.8)$ \\
\hline Venlafaxine & $0(0.0)$ & $9(0.9)$ & $9(0.9)$ \\
\hline Hydroxyzine & $4(0.4)$ & --- & $4(0.4)$ \\
\hline Buspirone & $0(0.0)$ & $5(0.5)$ & $5(0.5)$ \\
\hline Pregabalin & $0(0.0)$ & $0(0.0)$ & $0(0.0)$ \\
\hline Trifluoperazine & $0(0.0)$ & $0(0.0)$ & $0(0.0)$ \\
\hline Any of above & $392(40.2)$ & $345(35.4)$ & $607(62.3)$ \\
\hline
\end{tabular}

*All values are number of patients (\%); dose calculated using all values found in database

**Any use of long-acting benzodiazepines deemed potentially inappropriate

'Dose $>2 \mathrm{mg} / \mathrm{d}$ deemed potentially inappropriate

${ }^{2}$ Dose $>3 \mathrm{mg} / \mathrm{d}$ deemed potentially inappropriate

${ }^{3}$ Dose $>60 \mathrm{mg} / \mathrm{d}$ deemed potentially inappropriate

${ }^{4}$ Dose $>15 \mathrm{mg} / \mathrm{d}$ deemed potentially inappropriate

${ }^{5}$ Dose $>0.25 \mathrm{mg} / \mathrm{d}$ deemed potentially inappropriate

${ }^{6}$ Dose $>5 \mathrm{mg} / \mathrm{d}$ deemed potentially inappropriate

GAD: Generalized anxiety disorder 
the magnitude of potentially inappropriate prescribing in Germany among older patients with GAD. Further study is needed to ascertain the extent to which these other aspects of potentially inappropriate prescribing are evident in this patient population.

The clinical implication of our findings appears to be that benzodiazepines, amitriptyline, and doxepin are being overprescribed in Germany among elderly patients with GAD, especially among those with comorbid depression and/or sleep disorders. A major limitation of our study, however, is that we cannot assess the actual extent to which prescribing was truly clinically inappropriate. The treatment of GAD in the elderly is clinically complex and presents many challenges. Since we did not employ a "therapeutic timeline" as part of our research design, patients could have received potentially inappropriate agents either as initial treatment or following failure of safer, more appropriate agents. To the extent that the latter occurred, providers may have known about the risks associated with these medications and nonetheless used them only after a careful balancing of these risks against their potential benefits in patients with severe symptoms [29]. Further study is needed to clarify the extent of truly clinically inappropriate prescribing.

A second limitation of our study is that the list of potentially inappropriate agents may be too rigid. For example, while Beers classified amitriptyline as potentially inappropriate, Zhan et al. [13] deemed the use of low doses of this agent as appropriate in older adults in some instances, such as for the treatment of neuropathic pain. We note, however, that the Beers criteria are not disease-specific; these agents are deemed potentially inappropriate based exclusively on age $\geq 65$ years. Furthermore, the panel convened by Zhan et al. concluded that drugs such as amitriptyline "are often misused in clinical practice" [13].

Finally, we note that the database was limited to information from encounters with GPs. Patients who were seen for the treatment of GAD by psychiatrists may not have been included in our study (unless they also received a diagnosis of GAD from their GPs). Moreover, all information on prescription drugs is limited to those that are dispensed by GPs. The generalizability of our findings to all older patients with GAD is therefore unknown.

\section{Conclusion}

GPs in Germany often prescribe medications that have been designated as potentially inappropriate to their elderly patients with GAD - especially those with comorbid depressive disorders. Further research is needed to ascertain whether there are specific subgoups of elderly patients with GAD for whom the benefits of these medications outweigh their risks.

\section{Competing interests}

Funding for this research was provided by Pfizer Inc., New York, NY. Marko Mychaskiw, R.Ph., Ph.D., and Ellen Dukes, Ph.D., are employees of Pfizer Inc. as well as coauthors of this manuscript; they were involved with the design of the study, data analysis and interpretation, manuscript preparation, and publication decisions. Ariel Berger, M.P.H., John Edelsberg, M.D., M.P.H., and Gerry Oster, Ph.D., are employees of Policy Analysis, Inc., who were paid consultants to Pfizer in consultation with the development of this manuscript.

\section{Authors' contributions}

$\mathrm{AB}, \mathrm{MM}, \mathrm{ED}, \mathrm{JE}$, and GO all contributed to the conceptualization and design of study, data interpretation, and manuscript preparation. In addition, $A B, J E$, and $G O$ undertook all statistical analyses of the data. All authors read and approved the final manuscript.

\section{Acknowledgements}

$E D$ and MM, were both employees of Pfizer at the time the work was conducted. The analyses were conducted by AB, JE, and GO, employees of Policy Analysis, Inc. Policy Analysis, Inc received financial support from Pfizer, Inc. for the conduct of this analysis and development of this manuscript.

\section{References}

I. American Psychiatric Association: Diagnostic and Statistical Manual of Mental Disorders, Fourth Edition, Text Revision Washington DC, American Psychiatric Association; 2000.

2. Kessler R, Berglund $P$, Demler $O$, et al.: Lifetime prevalence and age of onset distributions of DSM-IV disorders in the National Comorbidity Survey Replication. Arch Gen Psychiatry 2005, 62:593-602.

3. Ormel J, VonKorff M, Ustun T, et al.: Common mental disorders and disability across cultures: Results from the WHO collaborative study on psychological problems in general health care. JAMA 1994, 272:1741-1748.

4. Wittchen HU, Kessler RC, Beesdo K, et al:: Generalized anxiety and depression in primary care: Prevalence, recognition, and management. J Clin Psychiatry 2002, 63(Suppl 8):24-34.

5. Baldwin DS, Anderson IM, Nutt DJ, et al.: Evidence-based guidelines for the pharmacological treatment of anxiety disorders: Recommendations from the British Association for Psychopharmacology. J Psychopharmacol 2005, 19:567-596.

6. Gorman JM: Treating generalized anxiety disorder. J Clin Psychiatry 2003, 64(Suppl 2):24-29.

7. Rickels K, Schweizer E: The spectrum of generalised anxiety in clinical practice: The role of short-term, intermittent treatment. BrJ Psych 1998, 34:49-54.

8. Shader RI, Greenblatt DJ: Use of benzodiazepines in anxiety disorders. N Engl J Med 1993, 328:|398-|405.

9. Oster G, Huse DM, Adams SF, et al.: Benzodiazepine tranquilizers and the risk of accidental injury. Am J Pub Health 1990, 90:1467-1470.

10. Beers $\mathrm{MH}$, Ouslander JG, Rollinger I, et al.: Explicit criteria for determining inappropriate medication use in nursing home residents. Arch Intern Med 1991, I 5 1:1825-1832.

II. Beers $\mathrm{MH}$ : Explicit criteria for determining potentially inappropriate medication use by the elderly: An update. Arch Intern Med 1997, 157:1531-1536.

12. Anderson GM, Beers MH, Kerluke K: Auditing prescription practice using explicit criteria and computerized drug benefit claims data. J Eval Clin Pract 1997, 3:283-294.

13. Field TS, Gurwitz JH, Glynn RJ, et al.: The renal effects of nonsteroidal anti-inflammatory drugs in older people: Findings from the Established Populations for Epidemiologic Studies of the Elderly. J Am Geriatr Soc 1999, 47:507-5 II. 
14. Fick DM, Cooper JW, Wade WE, et al:: Updating the Beers Criteria for potentially inappropriate medication use in older adults: Results of a US consensus panel of experts. Arch Intern Med 2003, 163:2716-2724.

15. Gurwitz JH, Avorn J: The ambiguous relation between aging and adverse drug reactions. Ann Intern Med I99/, I I 4:956-966.

16. Spore DL, Mor V, Larrat P, et al.: Inappropriate drug prescriptions for elderly residents of board and care facilities. $\mathrm{Am} J$ Public Health 1997, 87:404-409.

17. Aparasu RR, Mort JR: Inappropriate prescribing for the elderly: Beers criteria-based review. Ann Pharmacother 2000, 34:338-346.

18. Liu GG, Christensen DB: The continuing challenge of inappropriate prescribing in the elderly: An update of the evidence. J Am Pharm Assoc 2002, 42:847-857.

19. Caterino JM: Administration of inappropriate medications to elderly emergency department patients: Results of a national survey. Acad Emerg Med 2003, 10:493-494.

20. Willcox SM, Himmelstein DU, Woolhandler S: Inappropriate drug prescribing for the community-dwelling elderly. JAMA 1994, 272:292-296.

21. Fialova D, Topinkova E, Gambassi G, et al:: Potentially inappropriate medication use among elderly home care patients in Europe. JAMA 2005, 293: I348-I358.

22. Dietlein G, Schroder-Bernhardi D: Use of the Mediplus ${ }^{\circledR}$ patient database in healthcare research. Int J Clin Pharm Ther 2002, 40:130-133.

23. SAS® Proprietary Software, Release 8.4 SAS Institute Inc., Cary, NC.

24. Cumming RG, LeCouteur DG: Benzodiazepines and risk of hip fractures in older people: A review of the evidence. CNS Drugs 2003, I 7:825-837.

25. Petrovic M, Mariman A, Warie $\mathrm{H}$, et al:: Is there a rationale for prescription of benzodiazepines in the elderly? Review of the literature. Acta Clin Belg 2003. 58-27-36.

26. Gray SL, Lai KV, Larson EB: Drug-induced cognition disorders in the elderly: Incidence, prevention and management. Drug Saf 1999, 21:101-122.

27. Thomas RE: Benzodiazepine use and motor vehicle accidents. Systematic review of reported association. Can Fam Physician 1998, 44:799-808.

28. Gurwitz $\mathrm{J}$ : Suboptimal medication use in the elderly. The tip of the iceberg. JAMA 1994, 272:316-317.

29. AGS Panel on Persistent Pain in Older Persons. The management of persistent pain in older persons. J Am Geriatr Soc 2002, 50(Suppl 6):S205-S224.

30. Knight EL Avorn I: Quality indicators for appropriate medication use in vulnerable elders. Ann Intern Med 200I, 135:703-7I0.

31. Hamilton HJ, Gallagher PF, O'Mahony D: Inappropriate prescribing and adverse drug events in older people. BMC Geriatrics 2009, 9:5.

\section{Pre-publication history}

The pre-publication history for this paper can be accessed here:

http://www.biomedcentral.com/1471-2318/9/31/prepub
Publish with Biomed Central and every scientist can read your work free of charge

"BioMed Central will be the most significant development for disseminating the results of biomedical research in our lifetime. "

Sir Paul Nurse, Cancer Research UK

Your research papers will be:

- available free of charge to the entire biomedical community

- peer reviewed and published immediately upon acceptance

- cited in PubMed and archived on PubMed Central

- yours - you keep the copyright
BioMedcentral 